\title{
Implementación de ambientes virtuales de aprendizaje como estrategia motivadora para el desarrollo de competencias lectoras
}

\section{$\overline{\text { Implementation of virtual learning environments as a }}$ motivating strategy for the development of reading skills}

DOI: http://dx.doi.org/10.17981/cultedusoc.9.3.2018.28

Artículo de investigación. Fecha de recepción: 15/06/2018. Fecha de aceptación: 27/11/2018

Ewduin Cuello Alfaro';

Hernando Rivera Aconcha; Juan Arango Vanegas;

Jaime Jimenez Vides; Francisco Ruidiaz Moreno; Ruby Alfaro De Cuello;

Alicia Mansbach Torres; Nubia Cubides De Ballestas; Monica Torres Ruidiaz;

Juan Aranfo Vanegas; Osiris Toro Villalobos;Victoria Ruidiaz De Yepez;

Luis Yepez; Maria Avila Ricaurte; Delcy Camacho Castro; Iris Cortes Pedrozo;

Betty Lascarro Moya; Flor Ribon Moreno; Maria Lengua Muñoz;

Ena Trespalacios; Mildreth Villareal Oviedo; Lamar Cantillo Miranda; Maria

Saucedo Padilla; Nildre Ortega Borrego; Nilma Perez Alfaro;

Martha Hernandez Jimencez y Madel Mejia Estrada ${ }^{2}$

IED Néstor Andrés Rangel Alfaro (Colomnbia)

ewdincuello@gmail.com

Para citar este artículo:

Cuello. E., Rivera, H., Arango, J., Jimenez, J., Ruidiaz, F., Alfaro, R., Mansbach, A., Cubides, N., Torres, M., Aranfo, J., Toro, O., Ruidiaz, V., Yepez, L.,; Avila, M., Camacho, D., Cortes, I., Lascarro, B., Ribon, F., Lengua, M., Trespalacios, E., Villareal, M., Cantillo, L., Saucedo, M., Ortega, N., Perez, N., Hernandez, M. y Mejia, M. (2018). Implementación de ambientes virtuales de aprendizaje como estrategia motivadora para el desarrollo de competencias lectoras. Cultura. Educación y Sociedad 9(3), 247-254. DOI: http://dx.doi. org/10.17981/cultedusoc.9.3.2018.28

\section{Resumen}

El presente artículo presenta los resultados derivados del estudio Implementación de Ambientes virtuales de Aprendizaje como estrategia motivadora para el desarrollo de competencias lectoras. Además de analizar el uso de ambientes virtuales de aprendizaje como estrategia motivadora para el desarrollo de competencias lectoras, se proponen alternativas pertinentes para dinamizar los espacios de enseñanza-aprendizaje. La problemática general en las aulas de clases en torno al proceso de aprendizaje y en específico de las competencias lectoras de los estudiantes, promueve la búsqueda de caminos para el aprovechamiento y la estructuración de oportunidades para que el colectivo de docentes fortalezcna e innoven en sus prácticas pedagógicas y así generar mayor interés y motivación en ellos. La población objeto de estudio estuvo conformada por estudiantes de 9 grado de la Institución Educativa Néstor Andrés Rangel Alfaro. El estudio tuvo como propósito fomentar los ambientes virtuales de aprendizaje como una estrategia motivadora que fortalece el del desarrollo de las competencias lectoras.

Palabras clave: Ambientes virtuales de aprendizaje, comprensión lectora, educación básica.

\section{Abstract}

This article presents the results derived from the study Implementation of virtual learning environments as a motivating strategy for the development of reading skills; In addition to establishing how the use of virtual learning environments as a motivating strategy for the development of reading skills, it is relevant to boost the teaching-learning spaces. The general problems in the classroom around the learning process and specifically the reading skills of students, promotes the structuring of opportunities for teachers to strengthen and innovate in their pedagogical practices and thus generate greater interest and motivation in them. The population studied was made up of 9 th grade students from the Néstor Andrés Rangel Alfaro Educational Institution. The purpose of the study was to promote virtual learning environments as a motivating strategy that strengthens the development of reading skills.

Keywords: Virtual environments of learning, reading comprehension, basic education.

1Líder grupo de investigación.

2 Docentes IED Néstor Andrés Rangel Alfaro (Colomnbia)

- The author; licensee Universidad de la Costa - CUC. Cultura, Educación y Sociedad vol. 9 no. 3, pp. 247-254. Diciembre, 2018 Barranquilla. ISSN 2389-7724 Online 


\section{Introducción}

En el mundo de hoy el uso de las herramientas tecnológicas se ha convertido en una estrategia pertinente para dinamizar los espacios de enseñanza. La problemática general que se vive cotidianamente en las aulas de clases, en torno a los procesos de aprendizaje de los estudiantes, promueve la búsqueda de oportunidades para que el colectivo de docentes mejore y/o innoven sus prácticas de aula y haga evolucionar las rutinas que hasta ahora se han considerado suficientes.

Este estudio parte del fundamento de que el desarrollo de la capacidad lectora involucra una serie de procesos que el ser humano adquiere durante su desarrollo, los cuales son la base para adquirir conocimientos, mejorar su comunicación y ampliar su compresión del mundo. (Quintero, León y Pino, 2010). La UNESCO (2016), plantea que en la actualidad nos encontramos frente a una coyuntura histórica donde se cuenta con el desarrollo de gran cantidad de conocimiento, como también de espacio, estrategias, ambientes y herramientas que dan la accesibilidad al aprendizaje integral y de múltiples formas; pese a esto la educación atraviesa diversos afanes que logran verse desde diferentes perspectivas.

De un lado, la tecnología se ha convertido en un medio, un fin en sí misma, alejándose de su objetivo inicial y convirtiéndose en objeto de diversión, por encima de su función educativa; y por otro la asequibilidad de grupos humanos, con limitaciones de recursos económicos y limitaciones de acceso a las tecnologías aplicables a la educación, constituye una línea de distancia para que estas comunidades puedan integrarse en lo que implica globalización y de manera conjunta puedan salir del incesante círculo de la pobreza. En este sentido, Escobar, Pares y Barroso (2015), destacan la necesidad de fortalecer procesos forma- tivos y de aprendizaje desde la mediación de las tecnologías de la información y la comunicación, de manera que se garantice la compartición de información y conocimiento entre diferentes actores sociales.

Como plantea el Ministerio de Educación (2007): somos conscientes de que el aprendizaje, especialmente en los primeros años, deja una huella que perdura toda la vida; por lo tanto, debe ser una experiencia agradable, llena de sentido y significado, que no genere angustias frente a las equivocaciones, sino que sirvan para aprender y desarrollar un proceso de aprendizaje fortalecido con la lectura, utilizando las herramientas de las tecnologías de la información y la comunicación (TIC).

Consecuentemente, su desarrollo está vinculado con la creación, puesta en marcha y mejoramiento de ambientes grato de aprendizaje. En las TIC se privilegia la interacción comunicativa presencial y virtual que se promueva entre el docente y sus alumnos, se busca crear ambientes de aprendizaje que faciliten oportunidades a los alumnos, para que ellos desarrollen su propio conocimiento partiendo de su contexto y creen mayores saberes, desarrollen habilidades de pensamiento, capacidades, valores y actitudes. La construcción de un conocimiento de signo innovador por parte de los estudiantes, implica procesos de creación, socialización y adaptación. En este sentido, Vallejo (2011) resalta que los mecanismos de adaptación de los individuos contribuyen al fortalecimiento de valores, actitudes y formas de comportamiento que definen perfiles de desarrollo integral. Este planteamiento coincide con lo expuesto por Bravo, Marín y Carrera (2012), cuando asocian el concepto de integralidad a mecanismos de relacionamiento entre los actores sociales, con base en la compartición de conocimiento y generación de capacidades y capital social. 
Los referentes anteriores sirven de marco contextual para que el grupo de investigación conformado por docentes beneficiados con el programa Ciclón de la Institución Educativa Distrital (IED) Néstor Andrés Rangel Alfaro, identificaran que estas variables están asociadas al desarrollo de competencias en los estudiantes. Tal es el caso de las competencias lectoras se encunetra seriamente impactada con un bajo rendimiento que no solo se evidencia en el marco evaluativo institucional, si no que trasciende a los resultados de las Pruebas SABER.

Es pertinente señalar que la institución viene implementando como estrategia pedagógica el modelo constructivista, el cual de acuerdo lo expuesto por Hernández (2008), tiene un enfoque en la construcción del conocimiento, a partir de las diversas experiencias vivenciales ricas en contexto, aspecto que se ha visto fortalecido con la llegada de las nuevas tecnologías de la información, las cuales ofrecen un acceso amplio e ilimitado y permite direccionar el aprendizaje.

Según Batista (2006), un ambiente de aprendizaje está constituido como un espacio propicio para que los estudiantes aprovechen recursos informativos y medios didácticos para interactuar y realizar actividades, para el alcance de propósitos educativos establecidos; y a su vez cuando se hace referencia a la virtualidad de los mismos, se aluden entornos informáticos digitales e inmateriales que proveen las condiciones para la realización de actividades de aprendizaje

Por otro lado, Giroux (1997), expresa que la emergencia histórica de nuevos escenarios para la Pedagogía, mejorando los ambientes escolares tradicionales, se remontan a los años sesenta en Latinoamérica, con las experiencias educativas lideradas por comunidades e instituciones, con ideales liberacionistas en contextos de marginación, explotación económica y dominación política.
Es relevante manifestar que el crecimiento en la oferta y demanda de ambientes virtuales de aprendizaje revela que las entidades educativas han encontrado en las tecnologías de la información y comunicación un recurso valioso, y constituye una oportunidad frente a la mejora de la oferta en el contexto escolar (Herrera, 2002).

Sin embargo, a pesar de que los estudiantes tienen conocimientos previos, cabe destacar que se ha identificado que en gran proporción les resulta difícil comprender lo concerniente a las competencias relacionadas con la comprensión lectora; por lo tanto, se ha hecho evidente que es un factor que guarda estrecha relación en el proceso académico, investigativo y de aprendizaje en general.

Las competencias lectoras implican la identificación, comprensión y reflexión de la información encontrada en textos literarios y expositivos, asociados a distintas situaciones que pueden darse tanto dentro como fuera del contexto educativo, teniendo en cuenta los contenidos, estructura e intencionalidad de los mismos (ICFES, 2011; PISA, 2014).

El desarrollo de procesos para afianzar la comprensión lectora es fundamental para el aprendizaje significativo, en tanto que contribuye al buen desempeño en cualquier otra área de conocimiento (SEP, 2011). Sin este tipo de competencias lectoras no se puede alcanzar la comprensión y reflexión crítica de lo aprendido, por lo que es necesario que las escuelas no solo fomenten la lectura, sino que formen lectores competentes. En palabras de Moreno (2005, p. 156) "la escuela es responsable de los niños que no saben leer, no de los que no quieren leer".

En este sentido, las Tecnologías de la información y la comunicación (TIC) son herramientas útiles que permiten llevar a cabo prácticas y estrategias innovadoras que son de gran utilidad para fortalecer procesos de aprendizaje significativo en 
el contexto escolar (Ramírez, 2010), en este caso para fortalecer las competencias lectoras. Además, se ha demostrado que las estrategias basadas en las TIC incrementan la iniciativa y motivación de los estudiantes en relación a la apropiación de conocimiento frente a sus compromisos escolares (Avendaño y Martínez, 2013).

La UNESCO (2004), plantea que dentro del marco de los procesos educativos se deben llevar a cabo estrategias que promuevan la calidad de la educación mediante el uso de las TIC en la construcción de aprendizaje.

Si bien la enseñanza presencial es fundamental, acompañarla apropiadamente con el uso de la tecnología, impulsa al estudiante a enfrentar desafíos constantes que le llevarán a un conocimiento significativo. En este sentido, el uso de la tecnología permitiría eliminar la escuela tradicional y centrar el aprendizaje en el estudiante, haciéndolo más honesto con su aprendizaje, reflexivo y cuestionador de las prácticas, "pero lo más importante es que se deja de lado la memorización para pasar a la producción de conocimiento nuevo, incrementado por las experiencias de los estudiantes" (Cortes, Orozco, Luna y Martínez, 2015, p. 6).

Así mismo, para propiciar un espacio que realmente desarrolle competencias significativas en el contexto escolar, ha surgido la nueva propuesta de Ambiente virtual de Aprendizaje (AVA), el cual se concibe como "un proceso pedagógico (...) que genera condiciones, espacios interactivos, creativos, intencionados y lúdicos, donde se recrean circunstancias y se asumen roles que evocan sistemas de conocimiento, facilitando así su vivencia práctica" (SED, 2012, p.12).

\section{Metodología}

El artículo que se presenta es un producto derivado del estudio de la Implementación de ambientes virtuales de aprendizaje como estrategia motivadora para el desarrollo de competencia lectora; desarrollada en la Institución Educativa Néstor Andrés Rangel Alfaro; su objetivo principal ha sido dinamizar los espacios de enseñanzaaprendizaje.

El estudio se desarrolló con un abordaje metodológico cuantitativo, en el cual se realizó la contratación de la hipótesis desde los planteamientos argumentativos del marco teórico. Se seleccionó una muestra representativa de la población de estudio. El alcance del estudio fue de corte descriptivo, donde el propósito fue "medir o recoger información de manera independiente o conjunta sobre los conceptos o las variables a las que se refieren”. (Hernández, Collado \& Baptista, 2010, p.80) En este caso se buscó evaluar y fomentar ambientes virtuales de aprendizaje mediante el uso de TIC como estrategia motivadora al desarrollo de competencias lectoras.

Para la recolección de información, primeramente, se evaluaron los resultados de las Pruebas SABER (2016), de los estudiantes de $9^{\circ}$ en el componente de comprensión lectora. Luego, se diseñó y aplicó a los mismos una encuesta que buscaba indagar de qué manera los AVA y el uso de las TIC como herramientas motivadoras facilitan el desarrollo de competencias lectoras en ellos.

El instrumento tipo encuesta utilizado fue objeto de varios filtros, entre ellos la redacción y selección de preguntas, hasta la validación de la misma.

La aplicación de un instrumento tipo encuesta que pretendía medir el nivel de motivación e interés que generan la implementación de ambientes virtuales de aprendizaje y el uso de las tecnología de la información y comunicación, para el desarrollo de competencias lectoras en estudiantes, así mismo analizar las posibles dificultades que pueden presentar la población objetivo de estudio en la habilidad 
comunicativo-lectora. Los ítems fueron de carácter dicotómico, a excepción de uno abierto. Estos fueron las siguientes:

1. ¿Los docentes crean espacios para la lectura dentro o fuera del salón?

2. ¿Tienes computador en tu casa?

3. ¿Tus profesores utilizan herramientas virtuales hacia el aprendizaje de competencias lectoras?

4. ¿Conoces alguna plataforma virtual?

5. Si la respuesta anterior es SI, menciónala.

6. ¿Ha participado en el concurso Superat con el saber 2?0?

7. ¿Te ha parecido interesante el uso de esa herramienta virtual?

8. ¿El haber participado en supérate 2.0, ha fortalecido tu competencia Lectora?

\section{Resultados}

A partir del análisis de los principales hallazgos derivados del presente estudio se pueden identificar los siguientes resultados: En la Institución Educativa Departamental Néstor Andrés Rangel Alfaro, los docentes han manifestado su preocupación en tanto que la mayoría de los estudiantes muestran poco interés, apatía y desmotivación, para con el proceso de formación, lo cual genera dificultades para asimilar los procesos durante el desarrollo de las secuencias didácticas, especialmente en los procesos de comprensión lectora.

Los resultados de las pruebas Saber mostrados en la tabla 1 , en relación a la comprensión lectora en los estudiantes de noveno grado, se evidencian las dificultades de los estudiantes para construir un texto con relaciones de causalidad, reconocer datos explícitos e inferenciales, establecer relaciones de finalidad, encontrar la idea fundamental como contenido abstracto en textos informativos y literarios.

En la encuesta aplicada a los estudiantes, los resultados muestran, en la tabla 2 , que su percepción es positiva para el uso de herramientas virtuales en el desarrollo de las clases.

TABLA 1

Resultados Pruebas SABER, 2016. IED

Nestor Andrés Rangel Alfaro - Grado noveno

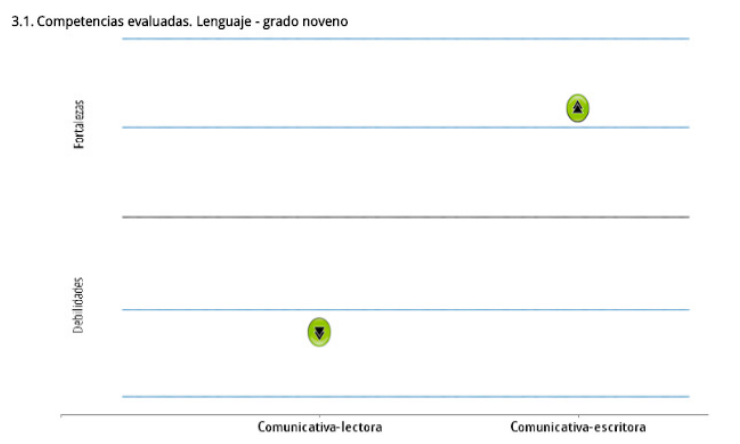

TABLA 2

Preguntas y resultados de encuesta realizada a estudiantes de $9^{\circ}$ Grado

\begin{tabular}{|c|c|c|}
\hline PREGUNTAS & SI & $\mathrm{NO}$ \\
\hline $\begin{array}{l}\text { ¿Los docentes crean } \\
\text { espacios para la lectura } \\
\text { dentro o fuera del salón? }\end{array}$ & 20 & 1 \\
\hline $\begin{array}{l}\text { ¿Tienes computador en } \\
\text { tu casa? }\end{array}$ & 6 & 15 \\
\hline $\begin{array}{l}\text { ¿Tus profesores utilizan } \\
\text { herramientas virtuales } \\
\text { hacia el aprendizaje de } \\
\text { competencias lectoras? }\end{array}$ & 18 & 3 \\
\hline $\begin{array}{l}\text { ¿Conoces alguna } \\
\text { plataforma virtual? }\end{array}$ & 21 & 0 \\
\hline $\begin{array}{l}\text { Si la respuesta anterior } \\
\text { es SI, menciónala. }\end{array}$ & $\begin{array}{l}\text { Supérate con el } \\
\text { saber. Colombia } \\
\text { Aprende. } \\
\text { Funtic. }\end{array}$ & \\
\hline $\begin{array}{l}\text { ¿Ha participado en el } \\
\text { concurso superar con el } \\
\text { saber } 2.0 ?\end{array}$ & 21 & 0 \\
\hline $\begin{array}{l}\text { ¿Te ha parecido } \\
\text { interesante el uso de esa } \\
\text { herramienta virtual? }\end{array}$ & 21 & 0 \\
\hline $\begin{array}{l}\text { ¿Al haber participado } \\
\text { en supérate } 2.0 \\
\text { ha fortalecido tu } \\
\text { competencia lectora? }\end{array}$ & 18 & 3 \\
\hline
\end{tabular}

Fuente: elaboración propia. 


\section{Conclusiones}

Los resultados derivados de la investigación realizada muestran que las pocas competencias lectoras presentes en estudiantes de la IED Néstor Andrés Rangel Alfaro representan una problemática que es necesario abordar en la práctica pedagógica de los docentes y en los procesos o experiencias de aprendizaje que se promueven en el aula.

A raíz de lo mencionado, surge la necesidad de replantearse el ejercicio de la práctica pedagógica apoyada en procesos investigativos y nuevas tecnologías de la información y comunicación, y dejar atrás las rutinas tradicionales, para darle cabida a la implementación de nuevas metas y prácticas innovadoras que posibiliten mayores y mejores resultados.

El problema no solamente se puede mirar desde una postura actual, es necesario también concebir los procesos lectores de manera adecuada, durante los primeros años de vida se deben instaurar bases sólidas en la habilidad para analizar y sintetizar los elementos fonéticos de la lengua, desarrollar una conciencia fonológica, lo cual constituye un predictor de éxito o fracaso en el aprendizaje de la lectura y es muy probable que lleguen a estar dentro del grupo de buenos lectores (Quintero, León \& Pino 2010).

Los espacios virtuales y el uso de las tecnologías de la información y la comunicación son una solución innovadora frente a la problemática mencionada, porque es una forma estratégica de servirse del acceso cotidiano de las tecnologías y, además, una herramienta que facilitará el dinamismo de los espacios académicos, lo que a su vez repercutirá en la motivación e interés de los estudiantes y, por tanto, con el acompañamiento adecuado por parte de los docentes. El estudiantado logrará desarrollar mejores competencias lectoras y su rendimiento global frente a los referentes estatales de evaluación proyectará mejores resultados.

Antes de la implementación de las herramientas TIC para el fortalecimiento de la lectura, también es importante explorar y descartar otras áreas de dificultades, como las manifiesta Ardila, Rosselli y Matute (2005), los cuales afirman que es necesario considerar otros problemas presentes en niños con dificultades en la lectura, carecen de habilidades de reconocimiento espacial y viso-perceptual: dificultad en movimientos de seguimiento y exploración visual, umbral de función visual disminuido, menor sensibilidad al movimiento y retraso al procesar letras visualmente. Algunos presentan defectos en el control de la convergencia ocular, fijación binocular inestable, fallas en el sentido de la direccionalidad visual y defectos en la percepción de profundidad.

Teniendo claro estos otros aspectos, sobre lo que hay que tener en cuenta para el desarrollo adecuado de la lectura, las herramientas tecnológicas son vistas como; facilitadoras de los procesos de aprendizaje, en donde: la motivación, el acompañamiento y el establecer las estrategias pertinentes, son fundamentales al momento de emprender este tipo de procesos en la lectura, las Tecnologías de la Información y la Comunicación (TIC) son actor fundamental y parte de la cultura académica en la que deben desarrollarse los docentes (Karam, Buitrago, Fagua y Romero,2013).

\section{Referencias}

Ardila, A., Rosselli, M. y Matute, E. (2005). Neuropsicología de los trastornos del aprendizaje. México, D.F.:. El Manual Moderno. 
Avendaño, I. y Martínez, D. (2013). Competencia lectora y el uso de las nuevas tecnologías de la información y comunicación. Revista Escenarios, 11(1), 7-22.

Bravo, O., Marín, F., Carrera, M. (2013). Redes inter-organizacionales y desarrollo local. Opción [en linea] , 29. Disponible en <http://www.redalyc. org/articulo.oa? id=31028677006> ISSN 1012-1587

Batista, M. (2006). Consideraciones para el diseño didáctico de ambientes virtuales de aprendizaje: una propuesta basada en las funciones cognitivas del aprendizaje. Revista Iberoamericana de Educación, 1-19.

Cortés, J., Orozco, G., Luna, V. y Martínez, A. (2015). El Aprendizaje Distribuido, primer paso a la virtualidad en la Enseñanza en el Nivel Universitario. Revista Iberoamericana de Producción Académica y Gestión Educativa.

Escobar, L., Paredes, A. y Barroso, M. (2015). Competencias del profesional de la información ante la evolución de las fuentes de información digitales. Accesbib: revista de bibliotecología y ciencias de la información, 4(1), 53-66. Recuperado de http://accesbib.blogspot.com/p/numero-actual.htm

Giroux, H. (1997). Cruzando límites. Trabajadores culturales, y políticas educativas. Barcelona, Paidós,

Hernández, S. (2008). El modelo constructivista con las nuevas tecnologías: Aplicado en el proceso de aprendizaje. RUSC. Universities and knowledge Society Journal, 5 (2), 26-35.

Hernández-Sampieri, R., FernándezCollado, C. y Baptista-Lucio, P. (2010). Metodología de la investigación. México, D.F.: Editorial Mc Graw Hill.
Herrera, L. (2002). Las fuentes del aprendizaje en ambientes educativos. Reencuentro. Análisis de problemas Universitarios, (35), 69-74.

Karam, J., Buitrago, A., Fagua, A. y Romero, Y. (2013). Estrategias de gamificación aplicadas al diagnóstico de la incorporación pedagógica de las TIC en una comunidad académica. Cultura, Educación y Sociedad 4(1), 55-74.

ICFES. (2011). Consulta de resultados individuales Saber 11. Icfes Interactivo: Consulta de resultados. Consultado en www.icfesinteractivo.gov.co/ resultadosSaber/resultadosSaber11/ res_individuales.htm

Moreno, V. (2005). Lectores competentes. Revista de Educación. 153-167

PISA. (2014). Programa Internacional de Evaluación de Estudiantes (Tercera ed.). Santiago, Chile: Agencia de Calidad de la Educación.

Quintero, A., León, A. y Pino, A. (2010). Conciencia fonológica y su relación con las dificultades de lectura. Cultura, Educación y Sociedad 2(1). 25-34.

Ramírez, C. (2010), Las TICS en el Aula. Granada: NNTT.

República de Colombia. Ministerio de Educación (2007). Foro Educativo Nacional. Bogotá, D.C.: MEN. Disponible en https://www.mineducacion. gov.co/1621/article-122265.html

SED. (2012). Aspectos que orientan la construcción y consolidación de los ambientes de aprendizaje. Bogotá, D.C.: Secretaría de Educación Distrital.

SEP. (2011). Manual de procedimiento para el fomento y la valoración de la competencia lectora en el Aula. México, D.F.: Secretaría de Educación Pública. 
UNESCO. (2004). Las tecnologías de la información y la comunicación en la formación docente. París: Ediciones UNESCO, División de Educación Superior.

UNESCO. (2016). Creación de futuros sostenibles para todos. La Educación al servicio de los pueblos y el planeta. Informe de seguimiento de la Educación en el mundo. París: Ediciones UNESCO.
Vallejo, O. (2011). Adaptación laboral: Factor clave para el rendimiento y la satisfacción en el trabajo. Cultura Educación y Sociedad, 2(1), 171 - 176. Recuperado de https://revistascientificas. cuc.edu.co/culturaeducacionysociedad/ article/view/946 